\title{
Evidências de Validade da Escala de Atitudes em Relação à Leitura: ERAS-Br
}

\author{
Lara Carolina de Almeida - Universidade do Estado do Rio de Janeiro, Rio de Janeiro, Brasil \\ Altemir José Gonçalves Barbosa - Universidade Federal de Juiz de Fora, Juiz de Fora, Brasil \\ Márcia Maria Peruzzi Elia da Mota - Universidade Salgado de Oliveira, Rio de Janeiro, Brasil
}

\begin{abstract}
Resumo
Para obter evidências de validade e fidedignidade, a Escala de Atitudes em Relação à Leitura (ERAS-Br) foi aplicada em 335 estudantes do Ensino Fundamental. Identificou-se que estruturas muldimensionais com dois (Leitura Acadêmica e Leitura Recreativa) ou três (Leitura Recreativa, Leitura Acadêmica Geral e Leitura Acadêmica Avaliação) fatores e um fator de segunda ordem são adequadas para a ERAS-Br. Tanto a medida como um todo quanto seus fatores apresentaram consistência interna satisfatória. Um procedimento de teste-reteste com um intervalo de dois anos e parte da amostra obteve coeficientes de estabilidade suficientes ou bons. De modo geral, as atitudes em relação à leitura tenderam a ser ligeiramente positivas, mas declinaram no intervalo de dois anos. Embora a ERAS-Br possua boas evidências de validade baseadas na estrutura interna e fidedignidade, o fato de as atitudes em relação à leitura se tornarem menos positivas ao longo dos anos é preocupante. Pesquisas adicionais são recomendadas.
\end{abstract}

Palavras-chave: atitudes, leitura, medidas, avaliação psicoeducacional

Evidence of the Validity of the Scale of Attitudes towards Reading: ERAS-Br

\begin{abstract}
To obtain evidence of validity and reliability, the Brazilian version of the Elementary Reading Attitude Survey (ERAS-Br) was applied to 335 elementary school students. It was identified that multidimensional structures with two (Academic Reading and Recreational Reading) or three (Recreational Reading, General Academic Reading and Academic Reading Assessment) factors and one factor of second order are suitable to the ERAS-Br. Both the measure as a whole and the factors showed a satisfactory internal consistency. A test-retest procedure with an interval of 2 years and part of the sample obtained sufficient or good stability coefficients. Overall, the attitudes toward reading tended to be slightly positive, but declined in the 2-year interval. Although the ERAS-Br has good validity evidence based on the internal structure and reliability, the fact that the attitudes toward reading become less positive over the years is worrying. Further studies are recommended.

Keywords: Attitudes; Reading; Measures; Psychoeducational Assessment
\end{abstract}

Las Evidencias de la Validez de la Escala de Actitudes hacia la Lectura: ERAS-Br

\begin{abstract}
Resumen
Para obtener evidencias de validez y confiabilidad, la Escala de Actitudes con relación a la Lectura (ERAS-Br) fue aplicada a 335 estudiantes de Enseñanza Primaria. Se identificó que estructuras multidimensionales con dos factores (Lectura Académica y Lectura Recreativa) o tres factores (Lectura Recreativa, Lectura Académica General y Lectura Académica de Evaluación) y con un factor de segundo orden, son adecuados para la ERAS-Br. Tanto la medida en su conjunto como sus factores presentaron consistencia interna satisfactoria. Un procedimiento de test-retest con un intervalo de dos años y parte de la muestra obtuvieron coeficientes de estabilidad suficientes o buenos. En general, las actitudes en relación a la lectura tendieron a ser ligeramente positivas, pero declinaron en el intervalo de dos años. Aunque la ERAS-Br presenta buenas evidencias de validez basadas en la estructura interna y confiabilidad, es preocupante el hecho de que las actitudes hacia la lectura se tornaron menos positivas a lo largo de los años. Se recomiendan investigaciones adicionales.

Palabras-clave: Actitudes; Lectura; Medidas; Evaluación Psicoeducativa.
\end{abstract}

Compreender o papel das atitudes em relação à leitura (AL) é importante por duas razões principais: (1) elas podem afetar o nível de habilidade em leitura, uma vez que influenciam o engajamento dos alunos; (2) AL negativas podem ocasionar a escolha por outras atividades que não a leitura, mesmo no caso de leitores fluentes (McKenna, Kear, \& Ellsworth, 1995). Assim, AL são construções psicológicas que desempenham um papel importante por moderarem o nível de motivação e a intenção de ler, bem como a relação entre as crenças individuais e as atividades de leitura (Petscher, 2010).

Há, de modo geral, concordância de que atitude é uma avaliação sumária de um objeto psicológico (Ajzen, 2001). Desde os primórdios do estudo desse construto, é consensual, também, que as atitudes são estados mentais que fazem com que as pessoas se aproximem ou se afastem de determinados objetos (Allport, 1935), ainda que exista um amplo debate relacionado ao 
quanto e como elas predizem comportamentos (Ajzen \& Fishbein, 2005). Fishbein e Ajzen (1975) destacam que existem três características básicas relacionadas a esse conceito: a atitude é aprendida, ela predispõe para a ação e as ações são consistentemente favoráveis ou desfavoráveis em direção ao objeto.

De acordo com o modelo triádico (Fishbein \& Ajzen, 1975), as atitudes são compostas por três dimensões: afeto, cognição e conação. $\mathrm{O}$ afeto diz respeito aos sentimentos e avaliações das pessoas em relação a um objeto, pessoa ou evento. A cognição denota aprendizado, crenças, opiniões e pensamentos em relação a um objeto. A conação se refere à intenção comportamental ou tendência para ação. Ressalta-se, com base nessa perspectiva, que as atitudes estão intimamente relacionadas à predisposição para se comportar de uma ou de outra maneira e, não necessariamente, com o comportamento em si.

As atitudes podem estar relacionadas a um incontável número de objetos. Ajzen (2001) assinala que, dependendo da perspectiva, um mesmo objeto pode suscitar diferentes atitudes, isso ocorre devido a avaliações e contextos diferentes que podem estar relacionados a ele. Dentre os múltiplos objetos das atitudes, este estudo elegeu, evidentemente, a leitura.

A mais antiga definição de AL parece ter sido proposta por Alexander e Filler (1976). Segundo ela, AL constituem um sistema de sentimentos relacionados à leitura que fazem com que o aprendiz procure ou evite atividades relacionadas a esse objeto. Os autores acrescentam, ainda, que essas atitudes variam de acordo com predisposições pessoais e são afetadas por variáveis internas ou relativas ao meio social.

Dentre os modelos de AL, optou-se, neste estudo, pelo de McKenna (McKenna et al., 1995). Para o autor, essas atitudes são, em grande parte, afetivas. Não obstante à primazia da dimensão afetiva, é possível perceber, também, a presença de componentes cognitivos e denotativos das AL no modelo de McKenna.

As AL podem ser avaliadas de diversas formas e com diferentes tipos de instrumentos. Dentre eles, destaca-se a Elementary Reading Attitude Survey - ERAS (McKenna \& Kear, 1990). Trata-se de uma escala de domínio público que tem sido amplamente usada nas pesquisas internacionais e apresentado propriedades psicométricas bastante satisfatórias.

McKenna e Kear (1990), no primeiro estudo com a ERAS, avaliaram 18138 alunos do primeiro ao sexto ano do Ensino Fundamental. Eles apresentaram, também, diversas evidências de validade de construto do instrumento, merecendo destaque as baseadas na estrutura interna, que identificou que a medida possui dois fatores: 1) Atitudes em Relação à Leitura Acadêmica (ALA) e 2) Atitudes em Relação à Leitura Recreativa (ALR). Para tanto, os autores realizaram uma análise fatorial exploratória (AFE). Inicialmente, obtiveram três fatores, sendo que nove dos 10 itens referentes à leitura acadêmica carregaram predominantemente em um fator, enquanto um item "acadêmico" (item 13 Anexo 1) apresentou cargas fatoriais equivalentes nos três fatores. $\mathrm{O}$ segundo fator foi composto por sete itens relacionados à leitura recreativa, e os outros três itens "recreativos" (6, 9 e 10) saturaram principalmente em um terceiro fator, mas também apresentam cargas fatoriais expressivas nos demais fatores. Com base nesses resultados, efetuaram uma segunda AFE programada para extrair somente dois fatores. Nessa análise, todos os itens, com uma exceção (item 13), saturaram significativamente nos fatores associados às ALR e ALA. Assim, os autores consideraram que a divisão em dois fatores é mais adequada para a escala e reflete dois aspectos distintos das atitudes em relação à leitura.

Além de McKenna e Kear (1990), parece que apenas Worrell, Roth e Gabelko (2007) também examinaram a estrutura fatorial da ERAS. Por meio de AFE, os autores extraíram inicialmente quatro fatores. Analisaram a estrutura fatorial com dois, três e quatro fatores e concluíram que o acréscimo do número de fatores não aumentaria expressivamente a variância explicada e baixaria o índice de consistência interna. Verificaram, ainda, que todos os 20 itens da ERAS carregaram bem, com cargas superiores a 0,35 , nos fatores do modelo original da escala, ou seja, ALA e ALR.

No estudo original da escala, McKenna e Kear (1990) identificaram boa consistência interna para os fatores da ERAS (ALR $-\alpha=0,82$. ALA $-\alpha=0,83$. Total $-\alpha=0,89)$. Ao considerar os anos escolares dos participantes, os autores obtiveram alfas de Cronbach entre 0,74 e 0,89 .

Outros estudos também analisaram a fidedignidade da ERAS (Tabela 1). De modo geral, eles analisam sua consistência interna com o alfa de Cronbach, atestando que se trata de uma medida fidedigna. Ressalta-se que, mais recentemente, as investigações sobre as propriedades psicométricas da escala são escassas, mas esse instrumento tem sido bastante utilizado em estudos experimentais ou quase-experimentais para avaliar possíveis efeitos de intervenções (p. ex., Bethea, 2012; Braden, 2012; Esteves \& Whitten, 2011; Hagan, 2012; Lee \& Yeo, 2014; Mihandoost \& Elias 2011; Newman, 


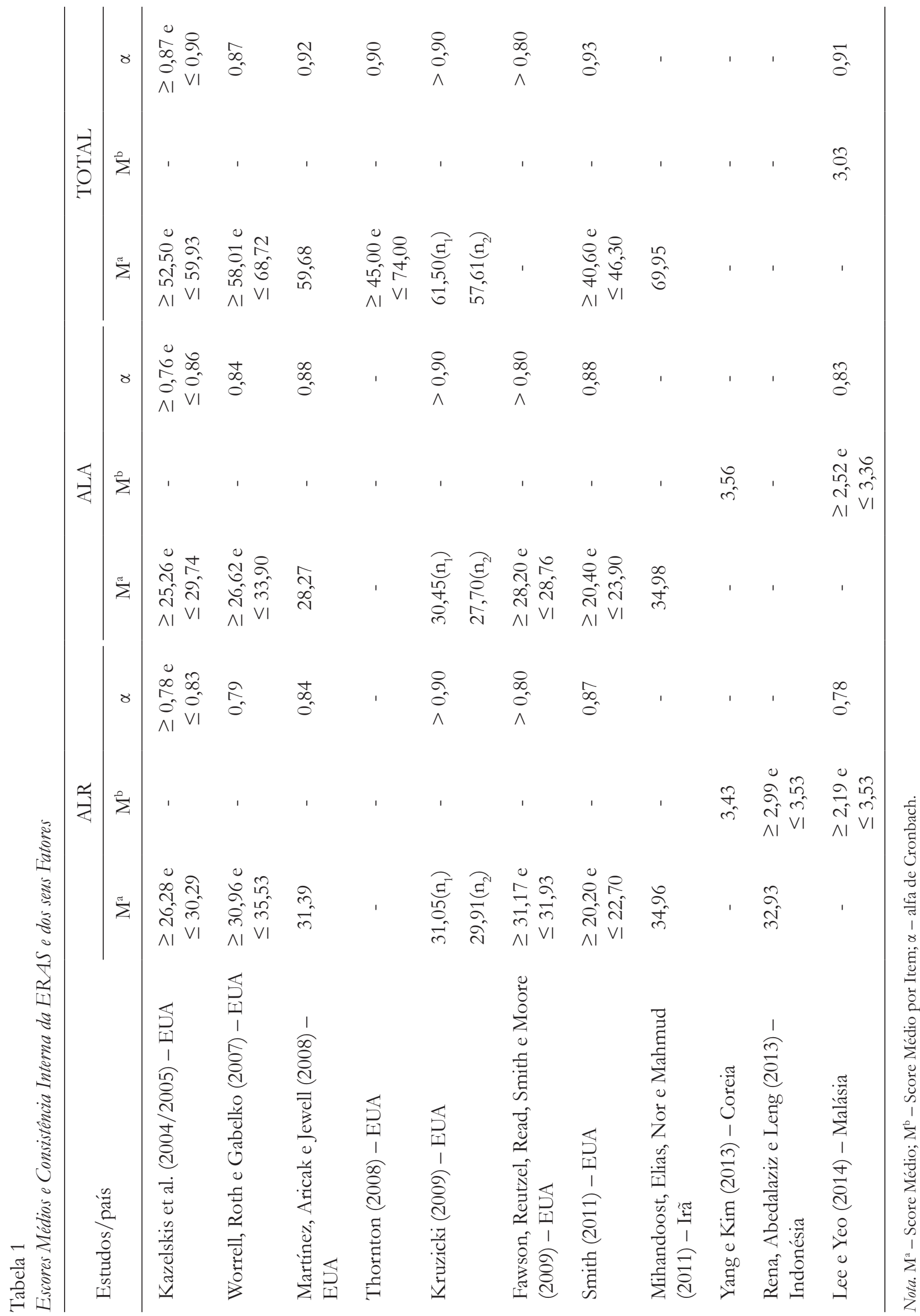


2013; Rena, Abedalaziz, \& Leng, 2013; Scheriff, 2012; Yang \& Kim, 2013).

Pesquisas que avaliam a estabilidade da ERAS são menos numerosas. Kush, Watkins, McAller e Edwards (1995) conduziram um estudo para verificar sua estabilidade num período de um ano. Os resultados reportados pelos autores indicaram estabilidade moderada nas atitudes das crianças nesse período de tempo tanto para as ALR e ALA quanto para o Total. No estudo de Kush e Watkins (1996), a escala não apresentou estabilidade significativa. Os autores constataram um considerável declínio nas AL em um intervalo mais longo - três anos - tanto para ALA quanto para ALR. Kazelskis et al. (2004/2005) verificaram que os coeficientes de estabilidade foram muito baixos, principalmente para os alunos do quarto e quinto anos, sugerindo considerável instabilidade nas AL em um período de uma semana.

Apesar da ampla literatura que relata pesquisas que empregaram a ERAS para avaliar atitudes em relação à leitura e de ela possuir versões em outros países [por exemplo, Irã (Mihandoost Elias, Nor, \& Mahmud, 2011), Coreia (Yang \& Kim, 2013), Indonésia (Rena Abedalaziz, \& Leng, 2013) e Malásia (Lee \& Yeo, 2014)], faltam estudos sobre sua estrutura fatorial e, principalmente, não há, ao que parece, uma versão em língua portuguesa dessa medida de AL. Assim, este artigo descreve estudos que buscaram evidências de validade e estimativas de fidedignidade para a versão da ERAS em língua portuguesa do Brasil (ERAS-Br). Especificamente, almejou-se analisar a consistência interna e a estabilidade da escala, bem como apresentar evidências de validade baseadas na estrutura interna da medida.

\section{Método}

\section{Participantes}

No total, foi empregada uma amostra não probabilística de 335 alunos de três escolas de uma cidade do interior de (informação retirada pela revista), sendo duas delas públicas e uma privada. Para obter evidências de fidedignidade do tipo teste-reteste, a amostra foi de 87 discentes, ou seja, aproximadamente $25 \%$ do total de participantes.

A diferença entre o total de participantes e a amostra do subgrupo teste-reteste foi decorrente, principalmente, do fato de que os estudantes do "teste" correspondem apenas àqueles que já tinham participado da amostra de um estudo anterior de Almeida (2011). As características demográficas das duas amostras são descritas na Tabela 2.

\section{Instrumentos}

\section{Questionário de Caracterização Demográfica.}

Esse instrumento foi desenvolvido pelos autores com o intuito de identificar a idade, a série, o tipo de escola (pública ou privada), o sexo e o número de reprovações dos participantes da pesquisa.

Escala de Atitudes em relação à Leitura (ERAS-Br). Trata-se da versão em língua portuguesa do Brasil da Elementary Reading Attitude Survey - ERAS (McKenna \& Kear, 1990). Esse instrumento passou

Tabela 2

Características Demográficas da Amostra Total e Teste-Reteste

\begin{tabular}{lccc}
\hline Variáveis & \multicolumn{1}{c}{ Amostra Total $^{\mathrm{a}}$} & Amostra Teste $^{\mathrm{b}}$ & Amostra Reteste $^{\mathrm{b}}$ \\
\hline Idade & $M=11,78 ; D P=1,83$ & $M=9,70 ; D P=1,72$ & $M=11,72 ; D P=1,78$ \\
Sexo & Feminino: $n=174 ; 51,94 \%$ & Feminino: $n=51 ; 58,62 \%$ & Feminino: $n=51 ; 58,62 \%$ \\
& Masculino: $n=161 ; 48,06 \%$ & Masculino: $n=36 ; 41,38 \%$ & Masculino: $n=36 ; 41,38 \%$ \\
Tipo de & Pública: $n=249 ; 74,33 \%$ & Pública: $n=63 ; 72,41 \%$ & Pública: $n=63 ; 72,41 \%$ \\
Escola & Privada: $n=86 ; 25,67 \%$ & Privada: $n=24 ; 27,59 \%$ & Privada: $n=24 ; 27,59 \%$ \\
Séries & $4^{\mathrm{o}}$ ano: $n=53 ; 15,82 \%$ & $2^{\mathrm{o}}$ ano: $n=13 ; 14,94 \%$ & $4^{\mathrm{o}}$ ano: $n=14 ; 16,09 \%$ \\
& $5^{\circ}$ ano: $n=50 ; 14,93 \%$ & $3^{\mathrm{o}}$ ano: $n=13 ; 14,94 \%$ & $5^{\circ}$ ano: $n=12 ; 13,79 \%$ \\
& $6^{\circ}$ ano: $n=49 ; 14,63 \%$ & $4^{\mathrm{o}}$ ano: $n=14 ; 16,09 \%$ & $6^{\circ}$ ano: $n=15 ; 17,24 \%$ \\
& $7^{\circ}$ ano: $n=59 ; 17,61 \%$ & $5^{\mathrm{o}}$ ano: $n=16 ; 18,39 \%$ & $7^{\circ}$ ano: $n=17 ; 19,54 \%$ \\
& $8^{\circ}$ ano: $n=76 ; 22,69 \%$ & $6^{\circ}$ ano: $n=19 ; 21,84 \%$ & $8^{\circ}$ ano: $n=17 ; 19,54 \%$ \\
& $9^{\circ}$ ano: $n=48 ; 14,33 \%$ & $7^{\circ}$ ano: $n=12 ; 13,79 \%$ & $9^{\circ}$ ano: $n=12 ; 13,79 \%$ \\
\hline
\end{tabular}

Nota. ${ }^{a} N=335 ;{ }^{\text {b }} N=87$. 
por um processo de adaptação equivalente ao proposto por Borsa, Damásio e Bandeira (2012), ou seja, tradução, síntese de traduções, avaliação por especialistas, tradução reversa, síntese de traduções reversas e estudo-piloto, dando origem à ERAS-Br (Anexo 1). Salienta-se, porém, que não bastou traduzir a medida; foi necessário, também, adaptar alguns itens para que ficassem adequados à realidade brasileira. Menciona-se, como exemplo disso, o item 7: "Como você se sente em relação a ler durante as férias do final de ano?". O original utiliza a expressão "summer vacation" (férias de verão) que, no Brasil, não é frequentemente usada. Assim, optou-se por substituí-la por "férias do final de ano".

Do mesmo modo que a escala original, a ERAS-Br avalia AL de estudantes, mais precisamente a dimensão afetiva (sentimentos). A medida é composta por 20 itens, sendo os dez primeiros referentes à leitura recreativa e os dez últimos à leitura acadêmica. Portanto, trata-se de uma medida bidimensional (ALR e ALA) com um fator de segunda ordem (total).

$\mathrm{O}$ instrumento possui quatro pontos de resposta, sendo que, para cada um dos 20 itens, o respondente deve escolher um ícone com uma expressão emocional. São apresentadas, evidentemente, quatro expressões emocionais distintas acompanhadas de rótulos que as descrevem na seguinte ordem: muito feliz, feliz, chateado e muito chateado. Ressalta-se que a escala original utiliza imagens do personagem de desenho animado Garfield para designar esses estados emocionais. No entanto, na adaptação para língua portuguesa, foram utilizados smiles. Tal mudança foi decorrente do fato de se buscar o uso de pontos de resposta menos viesados histórica e culturalmente.

Para computar os resultados da escala, a pontuação deve ser igual a quatro, três, dois ou um para, respectivamente, muito feliz, feliz, chateado e muito chateado. Em seguida, basta somar as respostas dos itens que compõem os fatores e, evidentemente, somar os dois fatores para chegar ao total da ERAS-Br. Desse modo, os escores das ALR e ALA podem variar entre 10 e 40 e o Total varia entre 20 e 80.

\section{Procedimento}

Após terem sido cumpridos os procedimentos éticos necessários, incluindo, por exemplo, aprovação por um Comitê de Ética (CAAE 0046.0.000.180-10 e CAAE 46293115.7.0000.5147), as coletas de dados foram efetuadas na própria escola onde os estudantes estavam matriculados. Salienta-se que, tanto na primeira coleta (Almeida, 2011) quanto na segunda, 24 meses após a primeira, foram adotados os mesmos procedimentos. Os participantes da pesquisa foram retirados em pequenos grupos (cinco a 15 alunos) das salas, durante o horário de aula, para a coleta de dados em uma sala anexa, silenciosa e livre de interrupções. Após as instruções serem dadas oralmente, a ERAS-Br e o questionário demográfico foram preenchidos individualmente. Nenhum participante apresentou dúvidas em relação aos itens da escala ou quanto ao segundo instrumento, precisando de cerca de 20 minutos para preencher ambos, demorando entre 10 e 15 minutos para completar a ERAS-Br.

A análise dos dados empregou tanto estatística descritiva (média, porcentagem etc.) quanto provas inferenciais. No último caso, o nível de significância foi de $5 \%$ por omissão. Foram realizadas análises fatoriais [AFE e análise fatorial confirmatória (AFC)], calculados o coeficiente de correlação intraclasse (CCI) e o alfa de Cronbach, bem como o teste $t$ para amostras pareadas.

A AFE foi realizada utilizando o método de Fatoração dos Eixos Principais e rotação oblimin, sendo retidas cargas fatoriais iguais ou superiores a 0,3 . Os métodos de fatoração e rotação foram escolhidos considerando, respectivamente, a distribuição dos dados e o pressuposto de que os fatores da ERAS-Br seriam relacionados (Damásio, 2012). Quanto à carga fatorial, teve-se como base Laros (2012), que propõe que valores iguais ou superiores ao estabelecido são significativos.

Foi realizada a análise fatorial confirmatória (AFC) com o software AMOS versão 20. Foram considerados os índices de qualidade do ajustamento dos modelos recomendados por Marôco (2010): razão do qui-quadrado pelos graus de liberdade (/gh); Goodness of Fit Index (GFI); Comparative Fit Index (CFI); Parsimony GFI (PGFI); Parsimony CFI (PCFI); Root Mean Square Error of Approximation (RMSEA); Expected Cross-Validation Index (ECVI).

\section{Resultados}

O escore médio do Total da ERAS-Br foi igual $58,82(D P=9,76)$. A Tabela 3 apresenta escores médios referentes aos itens da ERAS-Br. Ao analisar o pressuposto da normalidade, utilizando assimetria (skewness) e achatamento (kurtosis), foi possível perceber que ele é plausível, uma vez que os valores encontrados foram próximos de zero (Marôco, 2010) e menores que dois e sete, respectivamente (Curran, West, \& Finch, 1996).

Após constatar que os resultados do $\mathrm{KMO}(0,93)$ e do teste de Bartllet $\left(\chi_{0}^{2}=2610,95 ; g l=190 ; p<\right.$ 
0,001) atestavam a adequação da fatoração, realizou-se a AFE. Foram extraídos três fatores que explicam aproximadamente $44 \%$ da variância das respostas (Tabela 4). Ao analisar qualitativamente os itens que os compõem, os fatores foram designados Atitudes em relação à Leitura Acadêmica - Geral (ALAG), Atitudes em relação à Leitura Acadêmica - Avaliação (ALAA) e Atitudes em relação à Leitura Recreativa (ALR). Todos os itens apresentaram carga fatorial significativa $(>0,3)$.

A AFC (Tabela 5) testou dois modelos distintos oriundos, respectivamente, da teoria e da AFE. Baseado na teoria, o Modelo 1 mantém a estrutura fatorial da escala original em inglês, ou seja, dois fatores com dez itens e um fator (total) de segunda ordem. O Modelo 2 também possui um fator de segunda ordem, mas, a partir dos resultados da AFE, subdivide-se em três fatores: ALR com dez itens, ALAG com quatro itens e ALAA com seis itens.

Observa-se (Tabela 5) que tanto o Modelo 1 (bifatorial) quanto o Modelo 2 (trifatorial) apresentam bons índices de ajustamento. Nota-se, com base no ECVI, que o segundo apresentou uma qualidade de ajustamento um pouco melhor que o primeiro, corroborando o resultado da AFE. As cargas fatoriais dos itens do Modelo 1 variaram entre 0,45 (Item 19) e 0,75 (Item 7) e do Modelo 2 entre 0,54 (Item 18) e 0,75 (Item 7).

Ao considerar a amostra total e as duas estruturas fatoriais descritas, constatou-se que ALR apresentou um escore médio igual a 29,62 $(D P=5,58)$. Para ALA, ALAA e ALAG, as médias foram respectivamente $29,19(D P=5,06), 18,14(D P=3,28)$ e $11,05(D P=$ 2,40). Ao comparar os escores médios de ALR e ALA, não foi obtida diferença estatisticamente significativa $\left.\left[{ }_{0}(335 ; 334)=1,843 ; p=0,066\right)\right]$.

Quanto à fidedignidade da ERAS-Br, obteve-se um alfa de Cronbach igual a 0,91, sendo que excluir itens não elevaria a consistência interna da medida. Os alfas dos três fatores extraídos com a AFE (Tabela 4) podem ser classificados como apropriados. Ademais, observou-se que as correlações item-total podem ser classificadas como positivas e moderadas no caso dos

Tabela 3

Escores dos Itens da Escala de Atitudes em Relação à Leitura (ERAS-Br)

\begin{tabular}{lcccc}
\hline Itens & $M$ & $D P$ & Skewness & Kurtosis \\
\hline Ler num sábado chuvoso. & 2,79 & 0,80 & $-0,505$ & $-0,015$ \\
Ler durante o tempo livre. & 2,85 & 0,80 & $-0,449$ & $-0,109$ \\
Ler por lazer em casa. & 3,16 & 0,82 & $-0,861$ & 0,354 \\
Ganhar um livro de presente. & 3,37 & 0,76 & $-1,182$ & 1,173 \\
Gastar tempo livre lendo. & 2,76 & 0,81 & $-0,445$ & $-0,143$ \\
Começar a ler um novo livro. & 3,32 & 0,71 & $-0,958$ & 1,066 \\
Ler durante as férias de final de ano. & 2,49 & 0,96 & $-0,133$ & $-0,930$ \\
Ler ao invés de brincar. & 2,31 & 0,86 & 0,127 & $-0,643$ \\
Ir a uma livraria. & 3,35 & 0,70 & $-0,973$ & 1,003 \\
Ler diferentes tipos de livros. & 3,23 & 0,68 & $-0,728$ & 0,867 \\
Professor faz perguntas sobre o que leu. & 2,86 & 0,77 & $-0,557$ & 0,260 \\
Ler páginas de livros didáticos. & 2,51 & 0,77 & $-0,199$ & $-0,354$ \\
Ler na escola. & 2,99 & 0,73 & $-0,538$ & 0,374 \\
Ler livros escolares. & 2,70 & 0,83 & $-0,318$ & $-0,355$ \\
Aprender a partir de um livro. & 3,34 & 0,67 & $-0,901$ & 1,087 \\
Hora da leitura em sala de aula. & 3,03 & 0,77 & $-0,716$ & 0,521 \\
Histórias que lê nas aulas de português. & 3,04 & 0,78 & $-0,683$ & 0,345 \\
Ler em voz alta em sala de aula. & 2,92 & 0,92 & $-0,542$ & $-0,504$ \\
Usar um dicionário. & 2,80 & 0,79 & $-0,438$ & $-0,068$ \\
Fazer uma prova de leitura. & 2,99 & 0,88 & $-0,620$ & $-0,287$ \\
\hline
\end{tabular}


três fatores (Tabela 4) e da escala toda $(\geq 0,434$ e $\leq$ $0,676)$.

Ao analisar a consistência interna de cada um dos fatores da escala, considerando o Modelo 1, ou seja, a estrutura fatorial original da escala em inglês (ALA e ALR), obteve-se um alfa de Cronbach igual a 0,84 e 0,89 , respectivamente. Salienta-se que a exclusão de itens não elevaria a fidedignidade dos fatores. A correlação item-total com dois fatores foi, do mesmo modo que no caso de três fatores e da escala toda, positiva e moderada (ALR $-\geq 0,509$ e $\leq 0,707$. ALA $-\geq 0,432$ $\mathrm{e} \leq 0,601)$.

Obtiveram-se CCIs positivos moderados para o Total da ERAS-Br (Tabela 6), bem como para seus fatores independentemente de se adotar uma estrutura com dois (ALA e ALR) ou três (ALR, ALAA e ALAG) fatores. Ao comparar as médias do teste e do reteste da escala, observou-se que, excetuando-se ALR, as AL tenderam a se tornar negativas com o passar do tempo.

Tabela 4

Análise Fatorial Exploratória da ERAS-Br e Consistência Interna dos Fatores Extraídos

\begin{tabular}{|c|c|c|c|}
\hline ERAS & $\begin{array}{c}\text { Carga } \\
\text { Fatorial }\end{array}$ & $\begin{array}{l}\text { Alfa se o Item } \\
\text { for Excluído }\end{array}$ & $\begin{array}{l}\text { Correlação } \\
\text { Item-Total }\end{array}$ \\
\hline \multicolumn{4}{|l|}{ Fator 1 - Atitudes quanto à Leitura Recreativa } \\
\hline Gastar tempo livre lendo. & 0,827 & 0,869 & 0,707 \\
\hline Ler ao invés de brincar. & 0,723 & 0,877 & 0,602 \\
\hline Ler durante as férias de final de ano. & 0,674 & 0,869 & 0,699 \\
\hline Ir a uma livraria. & 0,595 & 0,876 & 0,613 \\
\hline Ler por lazer em casa. & 0,582 & 0,875 & 0,615 \\
\hline Ganhar um livro de presente. & 0,564 & 0,873 & 0,651 \\
\hline Ler num sábado chuvoso. & 0,555 & 0,876 & 0,599 \\
\hline Começar a ler um novo livro. & 0,555 & 0,877 & 0,593 \\
\hline Ler durante o tempo livre. & 0,524 & 0,875 & 0,615 \\
\hline Ler diferentes tipos de livros. & 0,387 & 0,882 & 0,509 \\
\hline Variância Explicada & $35,68 \%$ & - & - \\
\hline Alfa de Cronbach & 0,886 & - & - \\
\hline \multicolumn{4}{|c|}{ Fator 2 - Atitudes quanto à Leitura Acadêmica (Geral) } \\
\hline Ler livros escolares. & 0,751 & 0,660 & 0,606 \\
\hline Ler páginas de livros didáticos. & 0,610 & 0,682 & 0,569 \\
\hline Usar um dicionário. & 0,459 & 0,729 & 0,482 \\
\hline Histórias que lê nas aulas de português. & 0,457 & 0,701 & 0,534 \\
\hline Variância Explicada & $5,11 \%$ & - & - \\
\hline Alfa de Cronbach & 0,752 & - & - \\
\hline \multicolumn{4}{|c|}{ Fator 3 - Atitudes quanto à Leitura Acadêmica (Avaliação) } \\
\hline Ler em voz alta em sala de aula. & 0,572 & 0,748 & 0,513 \\
\hline Professor faz perguntas sobre o que leu. & 0,485 & 0,742 & 0,526 \\
\hline Hora da leitura em sala de aula. & 0,448 & 0,723 & 0,600 \\
\hline Ler na escola. & 0,436 & 0,738 & 0,546 \\
\hline Aprender a partir de um livro. & 0,356 & 0,755 & 0,474 \\
\hline Fazer uma prova de leitura. & 0,356 & 0,751 & 0,498 \\
\hline Variância Explicada & $2,89 \%$ & - & - \\
\hline Alfa de Cronbach & 0,776 & - & - \\
\hline
\end{tabular}


Tabela 5

Índices de Adequação dos Modelos Testados na AFC da ERAS-Br

\begin{tabular}{|c|c|c|c|}
\hline Índices & Valores de Referência & Modelo $1^{\mathrm{a}}$ & Modelo $2^{\text {b }}$ \\
\hline$\chi^{2} \mathrm{e} p$ & $\begin{array}{l}\text { Quanto menor melhor } \\
\qquad p<0.05\end{array}$ & $\begin{array}{c}\chi^{2}(165)=332,287 \\
p<0,001\end{array}$ & $\begin{array}{c}\chi^{2}(165)=317,766 \\
p<0,001\end{array}$ \\
\hline$\chi^{2} / g l$ & $\begin{array}{c}>5-\text { Ajustamento mal } \\
\text { ]2; 5] - Ajustamento sofrível } \\
\text { ]1;2] - Ajustamento bom } \\
\sim 1 \text { - Ajustamento muito bom }\end{array}$ & 2,014 & 1,926 \\
\hline CFI & $\begin{array}{c}<0.8-\text { Ajustamento mau } \\
{[0.8 ; 0.9[- \text { Ajustamento sofrível }}\end{array}$ & 0,933 & 0,938 \\
\hline GFI & $\begin{aligned} & {[0.9 ; 0,95[- \text { Ajustamento bom }} \\
\geq & 0.95-\text { Ajustamento muito bom }\end{aligned}$ & 0,911 & 0,914 \\
\hline PCFI & $\begin{array}{c}<0.6-\text { Ajustamento mau } \\
{[06 ; 0.8[- \text { Ajustamento bom }}\end{array}$ & 0,810 & 0,815 \\
\hline PGFI & $\geq 0.8-$ Ajustamento muito bom & 0,716 & 0,718 \\
\hline RMSEA e $p$ & $\begin{array}{c}\quad>0.10-\text { Inaceitável } \\
\text { ]0.05; } 0.10]- \text { Ajustamento bom } \\
\leq 0.05-\text { Ajustamento muito bom } \\
\\
\quad p \text {-value } \geq 0.05\end{array}$ & $\begin{array}{c}0,055 \\
p=0,160\end{array}$ & $\begin{array}{c}0,053 \\
p=0,299\end{array}$ \\
\hline ECVI & $\begin{array}{c}\text { Não especificado } \\
\text { Quanto menor, melhor }\end{array}$ & 1,264 & 1,221 \\
\hline
\end{tabular}

Nota. ${ }^{\mathrm{a} F i a b i l i d a d e}$ individual dos itens $\geq 0,21 \mathrm{e} \leq 0,56$.

${ }^{\mathrm{b}}$ Fiabilidade individual dos itens $\geq 0,30 \mathrm{e} \leq 0,56$.

Tabela 6

Evidências de Validade Teste-Reteste da ERAS-Br

\begin{tabular}{|c|c|c|c|c|}
\hline \multirow{2}{*}{ ERAS-Br } & \multicolumn{2}{|c|}{ Medidas } & \multirow{2}{*}{ CCI } & \multirow{2}{*}{ Teste $t^{a}$} \\
\hline & Teste & Reteste & & \\
\hline Total & $M=62,28(D P=8,54)$ & $M=59,95(D P=9,13)$ & 0,668 & $2,471 *$ \\
\hline ALA & $M=31,05(D P=4,95)$ & $M=29,61(D P=4,57)$ & 0,721 & $3,029 * *$ \\
\hline ALR & $M=31,23(D P=4,48)$ & $M=30,34(D P=5,57)$ & 0,622 & 1,576 \\
\hline ALAG & $M=11,96(D P=2,38)$ & $M=11,32(D P=2,21)$ & 0,648 & $2,529 *$ \\
\hline ALAA & $\mathrm{M}=19,10(\mathrm{DP}=3,01)$ & $\mathrm{M}=18,36(\mathrm{DP}=2,91)$ & 0,773 & $2,692 * *$ \\
\hline
\end{tabular}

Nota. ${ }^{a} g l=86 .{ }^{*} p<0,05 .{ }^{* *} p<0,00$.

\section{Discussão}

Ao considerar o escore médio do Total da ERAS -Br, é possível afirmar que ele é equivalente aos obtidos nos estudos de Kazelskis et al. (2004/2005), Worrell et al. (2007), Martínez, Aricak e Jewell (2008), Thornton
(2008) e Kruzicki (2009), sendo um pouco inferior ao de Mihandoost, Elias, Nor e Mahmud (2011) e superior ao de Smith (2011). A escala não possui pontos de corte, mas, se se considerar que a amplitude de sua mensuração varia entre 20 e 80 e que foi obtida uma média igual a 58,82 (DP =9,76), é possível afirmar que 
as AL tenderam à indefinição ou neutralidade. Se for adotado um ponto de vista mais otimista, elas podem ser consideradas ligeiramente positivas.

No caso dos escores médios dos itens, os resultados se assemelham aos discutidos no parágrafo anterior, pois a pontuação deles varia de um a quatro pontos e, ao estipular arbitrariamente um ponto médio (2,5 pontos), percebe-se que os alunos tiveram, no geral, atitudes neutras ou indefinidas em relação à leitura, ou seja, nem significativamente positivas, nem negativas $(\geq$ $2,31 \mathrm{e} \leq 3,37$ ). As poucas pesquisas internacionais (Lee \& Yeo, 2014; Rena et al. 2013; Yang \& Kim, 2013) que apresentam resultados para os itens da ERAS, tenderam a obter escores superiores aos encontrados no Brasil, denotando que as AL de estudantes brasileiros tendem a ser menos positivas que as dos malaios, indonésios e coreanos, por exemplo.

Não foram recuperados estudos que compararam os escores de ALA e ALR com estatística inferencial. Não obstante, parece que, do mesmo modo que na presente investigação, Mihandoost et al. (2011) e Smith (2011) também não detectaram diferença significativa entre os escores desses dois fatores. Discrepando dos resultados deste artigo, Fawson, Reutzel, Read, Smith e Moore (2009) identificaram que as primeiras tendem a ser mais positivas que as últimas e Lee e Yeo (2014) observaram que as ALR foram mais positivas que as ALA.

As ALA e ALR, como ocorreu com o Total, também tendem, evidentemente, a ser neutras ou levemente positivas na amostra avaliada. Resultado semelhante foi encontrado no estudo de Kazelskis et al. (2004/2005). Já as pesquisas de Mihandoost et al. (2011) e de Rena et al. (2013) obtiveram escores bem superiores, enquanto na de Smith (2011) os resultados foram inferiores. No que se refere às ALR, Worrell et al. (2007), Martínez et al. (2008), Kruzicki (2009) e Fawson et al. (2009) reportam resultados superiores aos encontrados na presente investigação. No caso das ALA, os escores foram semelhantes aos de Worrell et al. (2007) e de Kruzicki (2009), porém superiores aos de Martínez et al. (2008) e Fawson et al. (2009).

É importante frisar que a escala original (McKenna \& Kear, 1990) é bidimensional: ALR e ALA. Contudo, uma estrutura com três fatores parece ser mais adequada para a ERAS-Br, pois as ALA subdividiram-se em Leitura Acadêmica Geral (ALAG) e Leitura Acadêmica Avaliação (ALAA). Cabe ressaltar que outros estudos que analisaram a estrutura fatorial da ERAS (McKenna \& Kear, 1990; Worrell et al., 2007) por meio de AFE também extraíram mais de dois fatores, mas optaram por manter a estrutura inicialmente proposta para ela.

Não foram encontrados estudos que fizeram AFC da ERAS. Reitera-se que, na presente investigação, dois modelos foram testados: Modelo 1 - estrutura bidimensional original (ALA e ALR); e Modelo 2 - ALR, ALAA e ALAG. Segundo Marôco (2010), todos os itens da escala devem ter pesos fatoriais iguais ou superiores a 0,5 para atestar a validade fatorial, o que não pôde ser observado no Modelo 1. O autor assevera, ainda, que o valor apropriado para a fiabilidade individual dos itens deve ser maior ou igual a 0,25 , o que ocorre no Modelo 2, mas não no Modelo 1. Os índices de ajustamento também evidenciaram que o Modelo 2 é um pouco mais apropriado. Entretanto, esses resultados não parecem ser suficientes para que se proponha uma estrutura fatorial para a ERAS-Br distinta da original. Antes há que se realizarem mais estudos com amostras quantitativa e qualitativamente superiores às desta pesquisa.

A consistência interna da ERAS-Br pode ser considerada satisfatória e é equivalente à obtida em outros estudos que fizeram uso da ERAS. Para a escala toda, o Alfa de Cronbach foi semelhante aos das investigações de McKenna e Kear (1990), Martínez et al. (2008), Thornton (2008), Kruzicki (2009), Smith (2011) e Lee e Yeo (2014) e superior ao reportado por Kazelskis et al. (2004/2005), Worrell et al. (2007) e Fawson et al. (2009). Ao considerar apenas os itens que avaliam ALA, a consistência interna também foi adequada e equivalente à obtida por McKenna e Kear (1990), Worrell et al. (2007), Fawson et al. (2009) e Lee e Yeo (2014). No caso das ALR, o alfa de Cronbach também foi adequado e, ainda, superior ao apresentado por McKenna e Kear (1990), Worrell et al. (2007), Martínez et al. (2008), Fawson et al. (2009), Smith (2011) e Lee e Yeo (2014). Portanto, a ERAS-Br possui uma boa consistência interna e pode ser considerada uma medida fiável.

Constatou-se que, num período de dois anos, a ERAS-Br apresentou coeficientes de estabilidade que variaram entre suficientes (Total, ALR e ALAG) e bons (ALA e ALAA). Ao efetuar um estudo de teste-reteste da ERAS com intervalo de um ano, Kush et al. (1995) identificaram um coeficiente $(0,43)$ que foi classificado como "estável". Kazelskis et al. (2004/2005) e Kush e Watkins (1996) não obtiveram os mesmos resultados, no primeiro caso as crianças foram avaliadas em um período curto - uma semana - e no segundo caso em um período longo - três anos - e ambos constataram instabilidade tanto para as ALA quanto para as ALR. 
Desse modo, há que se questionar o quanto as atitudes são ou não estáveis ao longo do tempo. O modelo triádico de atitudes (Fishbein \& Ajzen, 1975) dá indícios de que esse constructo não é, necessariamente, estável, uma vez que elas influenciam e são influenciadas por sentimentos, avaliações, aprendizado, opiniões e pensamentos. No caso específico das AL, Alexander e Filler (1976) salientam o quanto o seu desenvolvimento é suscetível a influências sociais, culturais, acadêmicas e pessoais. Portanto, parece não ser sensato esperar que elas sejam tão estáveis quanto, por exemplo, personalidade e inteligência.

Observou-se, por fim, que as ALA e as AL (Total) tenderam, com o passar do tempo, a se tornarem menos positivas. Kush e Watkins (1996) também constataram declínio, mais especificamente para ALA, ALR e evidentemente o Total, no intervalo de três anos. Kazelskis et al. (2004/2005), apesar de não especificarem o quanto as atitudes declinaram ao longo de uma semana, também observaram resultados mais negativos para ALA, ALR e Total na segunda avaliação. McKenna, Kear e Ellsworth (1995) identificaram, em um estudo transversal, que as AL tendem a se tornar cada vez mais negativas ao longo dos anos escolares. As constatações da presente pesquisa e das citadas são alarmantes, pois o declínio das AL pode fazer com que os estudantes se tornem leitores menos proficientes ou, pelo menos, não progridam, acabando por se afastarem desse tipo de atividade. É preciso considerar, ademais, que o declínio das ALA observado neste estudo parece estar diretamente relacionado aos problemas do sistema educacional brasileiro, já que elas são, como a própria designação evidencia, estreitamente influenciadas pela escola.

Os resultados da presente investigação precisam ser considerados com cautela, uma vez que se trata do primeiro estudo sobre a ERAS-Br. Outras pesquisas utilizando o mesmo instrumento e contando com amostras maiores e mais diversificadas são aconselhadas. Recomendam-se, mais especificamente, análises de sua estrutura fatorial, pois a AFE e a AFC aqui apresentadas fizeram uso da mesma amostra, o que é questionável (Laros, 2012), obtenção de evidências de validade para a escala baseadas na relação com outras variáveis (p.ex., desempenho em leitura) e investigações sobre variáveis associadas ao declínio das AL ao longo do tempo, já que as conhecer é fundamental para o desenvolvimento e a implantação de intervenções em ambientes escolares que almejem promover o engajamento dos estudantes em atividades de leitura.

\section{Referências}

Ajzen, I. (2001). Nature and operation of attitudes. $A n$ nual Review of Psychology, 52, 27-58, doi: 10.1146/ annurev.psych.52.1.27

Ajzen, I., \& Fishbein, M. (2005). The influence of attitudes on behavior. Em D. Albarracín, B. T. Johnson \& M. P. Zanna (Eds.), The handbook of attitudes (pp. 173-221). Mahwah, NJ: Erlbaum.

Alexander, J. E., \& Filler, R. C. (1976). Attitudes and reading. Newark, DE: International Reading Association.

Allport, G. W. (1935). Attitudes. Em C. Murchison (Ed.), Handbood of social psychology (pp. 798-844). Worcester, MA: Clark University Press.

Almeida. L. C. (2011). Leitor talentoso: identificação e preditores. Dissertação de Mestrado, Universidade Federal de Juiz de Fora, Juiz de Fora, Minas Gerais.

Bethea, S. L. (2012). The impact of Oakland freedom school's summer youth program on the psychosocial development of african american youth. Journal of Black Psychology, 38(4), 442-454, doi: 10.1177/0095798411431982.

Borsa, J. C., Damásio, B. F., \& Bandeira, D. R. (2012). Adaptação e validação de instrumentos psicológicos entre culturas: Algumas considerações. Paidéia, 22, 423-432, doi: 10.1590/1982-43272253201314.

Braden, M. J. (2012). Impacting attitudes towards reading in the second grade classroom: a reading role model intervention. Master thesis, Wichita State University, Wichita, Kansas.

Curran, P. J., West, S. G., \& Finch, J. F. (1996). The robustness of test statistics to nonnormality and specification error in confirmatory factor analysis. Psychological methods, 1(1), 16-29, doi: 10.1037/1082-989X.1.1.16

Damásio, B. F. (2012). Uso da análise fatorial exploratória em psicologia. Avaliação psicológica, 11(2), 213-228. Recuperado de http://pepsic. bvsalud.org/scielo.php? script $=$ sci_arttext\&pi$\mathrm{d}=$ S1677-04712012000200007\&lng=pt\&tlng=pt

Esteves, K. J., \& Whitten, E. (2011). Assisted reading with digital audiobooks for students with reading disabilities. Reading Horizons, 51(1), 21-40. Recuperado de http://scholarworks.wmich.edu/ reading_horizons/vol51/iss1/4

Psico-USF, Bragança Paulista, v. 22, n. 3, p. 485-500, set./ der. 2017 
Fawson, P. C., Reutzel, D. R., Read, S., Smith, J. A., \& Moore, S. A. (2009). The influence of differing the paths to an incentive on third graders' reading achievement and attitudes. Reading Psychology, 30(6), 564-583, doi: 10.1080/02702710902753954.

Fishbein, M., \& Ajzen, I. (1975). Belief, attitude, intention, and behavior: An introduction to theory and research. Reading, MA: Addison-Wesley.

Hagan, E. (2013). Student reading attitudes in relation to the instrucional approach. (Tese de doutorado). Department of Education Leadership, Northwest Missouri State University, Maryville, Missouri.

Kazelskis, R., Thames, D., Reeves, C. Flynn, R., Taylor, L., Beard, L. A., \& Turnbo, D. (2004/2005). Reliability and stability of elementary reading attitude survey (ERAS) scores across gender, race, and grade level. The Professional Educator, 27(1/2), 29-37. Recuperado de http:/ / files.eric.ed.gov/fulltext/EJ728479.pdf

Kruzicki, H. A. (2009). The effects of cross-age reading on upper elementary students'attitudes toward reading. (Tese de doutorado). College of Liberal Studies Psychology, University of Wisconsin-La Crosse, Wisconsin.

Kush, J. C., \& Watkins, M. W. (1996). Long- term stability of children's attitudes toward reading. Journal of Educational Psychology, 89, 315-319, doi: 10.1080/00220671.1996.9941333.

Kush, J. C., Watkins, M. W., McAller M. T., \& Edwards V. A. (1995). One-year stability of the elementary reading attitude survey. Mid-Western Educational Researcher, 8(1), 11-14. Recuperado de http://edpsychassociates.com/Papers/ ERASshort(1995).pdf

Laros, J. A. (2012). O uso da análise fatorial: Algumas diretrizes para pesquisadores. In: L. Pasquali (Ed.), Análise Fatorial para pesquisadores (pp. 141-160). Brasília: LabPAM.

Lee, M., \& Yeo, K. (2014). Influence of home literacy environment on children reading attitude. Journal of Education and Practice, 5(8), 119-138. Recuperado de http://www.iiste.org/Journals/index.php/ JEP/article/viewFile/11648/11991

Marôco, J. (2010). Análise de equações estruturais: Fundamentos teóricos, software \& aplicações. Pêro Pinheiro: ReportNumber.

Martínez, R. S., Aricak, O. T., \& Jewell, J. (2008). Influence of reading attitude on reading achievement:
A test of the temporal-interaction model. Psychology in the Schools, 45(10), 1010-1022, doi: 10.1002/ pits. 20348 .

McKenna, M. C., \& Kear, D. J. (1990). Measuring attitude toward reading: A new tool for teachers. The Reading Teacher, 43(8), 626-639, doi: 10.1598/ RT.43.8.3

McKenna, M., Kear, D., \& Ellsworth, R. A. (1995). Children's attitudes toward reading: A national survey. Reading Research Quarterly, 30, 934-956, doi: 10.2307/748205.

Mihandoost, Z., \& Elias, H. (2011). The effectiveness of the barton's intervention program on reading comprehension and reading attitude of students with dyslexia. Iranian Journal of Psychiatry and Behavioral Sciences, 5(2), 43-52. Recuperado de http:/ / mazums.ac.ir/dorsapax/Data/Sub_15/File/ etoolsfile1Mihandoost-104.pdf

Mihandoost, Z., Elias, H., Nor, S., \& Mahmud, R. (2011). A comparison of the reading motivation and reading attitude of students with dyslexia and students without dyslexia in the elementary schools in Ilam, Iran. International Journal of Psychological Studies, 3(1), 17-27. Recuperado de http:// www.ccsenet.org/journal/ index.php/ijps/issue/ view/350

Newman, K. (2013). Time-on-task during independent reading: The effect of the use of ipads on student accountability and time-on-task. Doctoral dissertation, University of Wisconsin-River Falls, Wisconsin.

Petscher, Y. (2010). A meta-analysis of the relationship between student attitudes towards reading and achievement in reading. Journal of Research in Reading, 33(4), 335-355, doi: 10.1111/j.1467-9817.2009.01418.x

Rena, S., Abedalaziz, N., \& Leng, C. H. (2013). The relationship between parenting styles and students' attitude toward leisure time reading. The Malaysian Online Journal of Educational Science, 1(2), 37-54. Recuperado de http://moj-es.net/volume/mojesvolume01-i02.pdf\#page $=45$

Scheriff, T. J. N. (2012). The effects of repeated readings on third grade students'reading achievement and attitudes (Tese de doutorado). Liberty University, Lynchburg, Virginia. 
Smith, D. M. (2011). Readers theatre: Its effectiveness in improving reading fluency, student motivation, and attitudes toward reading among second-grade students. (Tese de doutorado). School Psychology, The Pennsylvania State University.

Thornton, G. P. (2008). Effects of modified fluency-oriented reading instruction on african american boys'oral reading fluency and attitudes toward reading. (Tese de doutorado). Auburn University, Auburn, Alabama.

Worrell, F. C., Roth, D. A., \& Gabelko, N. H. (2007). Elementary reading attitude survey (ERAS) scores in academically talented students. Roeper Review, 29(2), 119-124, doi: 10.1080/02783190709554395

Yang, M. S., \& Kim, J. K. (2013). The study of the relationship among reading trend, reading motivation, reading attitude, and self-directed learning. $A d$ vanced Science and Technology Letters, 36, 83-88, doi: 10.14257/astl.2013.36.19

Recebido em: 22/03/2016

Reformulado em: 23/05/2016; 01/06/2016

Aprovado em: 07/11/2016 


\section{Anexo 1 - ERAS-Br}

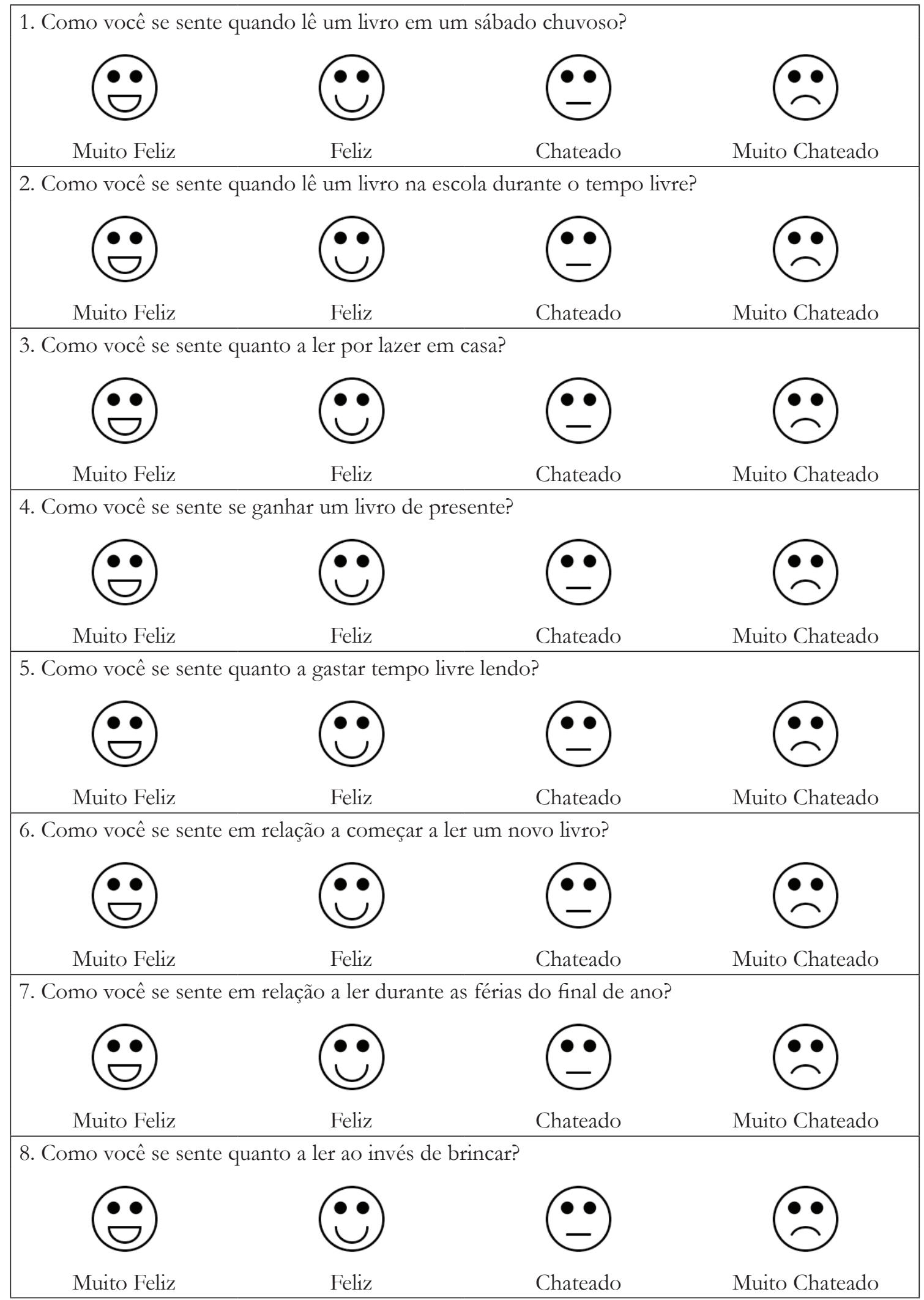




\begin{tabular}{|c|c|c|c|}
\hline \multicolumn{4}{|c|}{ 9. Como você se sente em relação a ir a uma livraria? } \\
\hline Muito Feliz & Feliz & Chateado & Muito Chateado \\
\hline \multicolumn{4}{|c|}{ 10. Como você se sente quanto à leitura de diferentes tipos de livro? } \\
\hline Muito Feliz & Feliz & Chateado & Muito Chateado \\
\hline \multicolumn{4}{|c|}{ 11. Como você se sente quando o(a) professor(a) faz perguntas sobre o que você leu? } \\
\hline Muito Feliz & Feliz & Chateado & Muito Chateado \\
\hline \multicolumn{4}{|c|}{ 12. Como você se sente quanto a ler páginas de livros didáticos e lições? } \\
\hline Muito Feliz & Feliz & Chateado & Muito Chateado \\
\hline \multicolumn{4}{|c|}{ 13. Como você se sente sobre ler na escola? } \\
\hline Muito Feliz & Feliz & Chateado & Muito Chateado \\
\hline \multicolumn{4}{|c|}{ 14. Como você se sente sobre ler seus livros escolares? } \\
\hline Muito Feliz & Feliz & Chateado & Muito Chateado \\
\hline \multicolumn{4}{|c|}{ 15. Como você se sente quanto a aprender a partir de um livro? } \\
\hline Muito Feliz & Feliz & Chateado & Muito Chateado \\
\hline \multicolumn{4}{|c|}{ 16. Como você se sente quando é a hora da leitura em sala de aula? } \\
\hline Muito Feliz & Feliz & Chateado & Muito Chateado \\
\hline
\end{tabular}




17. Como você se sente em relação às histórias que lê nas aulas de leitura ou português?


Sobre os autores:

Lara Carolina de Almeida é psicóloga, mestre em Psicologia pela Universidade Federal de Juiz de Fora e doutoranda do Programa de Pós-Graduação em Psicologia Social da Universidade do Estado do Rio de Janeiro. Atualmente atua como psicóloga clínica.

E-mail: lara.karol@hotmail.com

Altemir José Gonçalves Barbosa é psicólogo, mestre em Psicologia Escolar (PUC -Campinas), doutor em Psicologia (PUC - Campinas), professor do Departamento de Psicologia e do Programa de Pós-Graduação em Psicologia da Universidade Federal de Juiz de Fora (UFJF) e bolsista produtividade do CNPq.

E-mail:altgonc@gmail.com

Márcia Maria Peruzzi Elia da Mota é doutora em Psicologia (University of Oxford), diretora do Instituto de Psicologia da UERJ, professora titular do Programa de Pós-Graduação em Psicologia da Universidade Salgado de Oliveira e bolsista de produtividade nível 2 do CNPq.

E-mail:mmotapsi@gmail.com

Contato com os autores:

Universidade Federal de Juiz de Fora

Rua José Lourenço Kelmer, s/n - Campus Universitário

Bairro São Pedro

Juiz de Fora-MG, Brasil

CEP: 36036-900 